\title{
Heart rate as marker of successful percutaneous renal denervation
}

\author{
Cédric Lehner, Stéphane Cook, Mario Togni, Jean-Jacques Goy \\ Department of Cardiology, Fribourg, University Hospital, Switzerland
}

\begin{abstract}
A 52-year-old man with long-standing resistant essential hypertension, inappropriate sinus tachycardia and known ischaemic heart disease was referred to our centre for percutaneous renal denervation. The arterial hypertension was treated for six years and multiple drug combinations (angiotensin-converting-enzyme inhibitors, angiotensin receptor blockers, calcium antagonist, $\beta$-blockers, diuretics and spironolactone) were tried in the past. His current therapy included meto-
\end{abstract}

prolol succinate $200 \mathrm{mg} /$ day, perindopril $5 \mathrm{mg} /$ day, doxazosin mesylate $4 \mathrm{mg} /$ day and ivabradine $7.5 \mathrm{mg}$ twice a day. Diuretic treatment had been used but recently stopped due to patient intolerance. His baseline 24-hour recordings are depicted in figure 1, panel A, and showed a mean blood pressure of 137/96 mm Hg and a mean heart rate was $96 \mathrm{bpm}$.

The procedure was performed in accordance with the manufacturer protocol, under general anaesthesia.

Figure 1

A Baseline 24-hour recordings with mean blood pressure of 137/96 mm Hg and mean heart rate of $96 \mathrm{bpm}$.

B Bilateral renal angiograms prior (1 and 2) and after (3 and 4) the endovascular procedure. Post-procedural renal angiograms demonstrated ablation's nodes (K).

C Two weeks after the procedure, 24-hour recordings demonstrating a significant drop in mean blood pressure at $124 / 85 \mathrm{~mm} \mathrm{Hg}$ and in mean heart rate at $75 \mathrm{bpm}$.

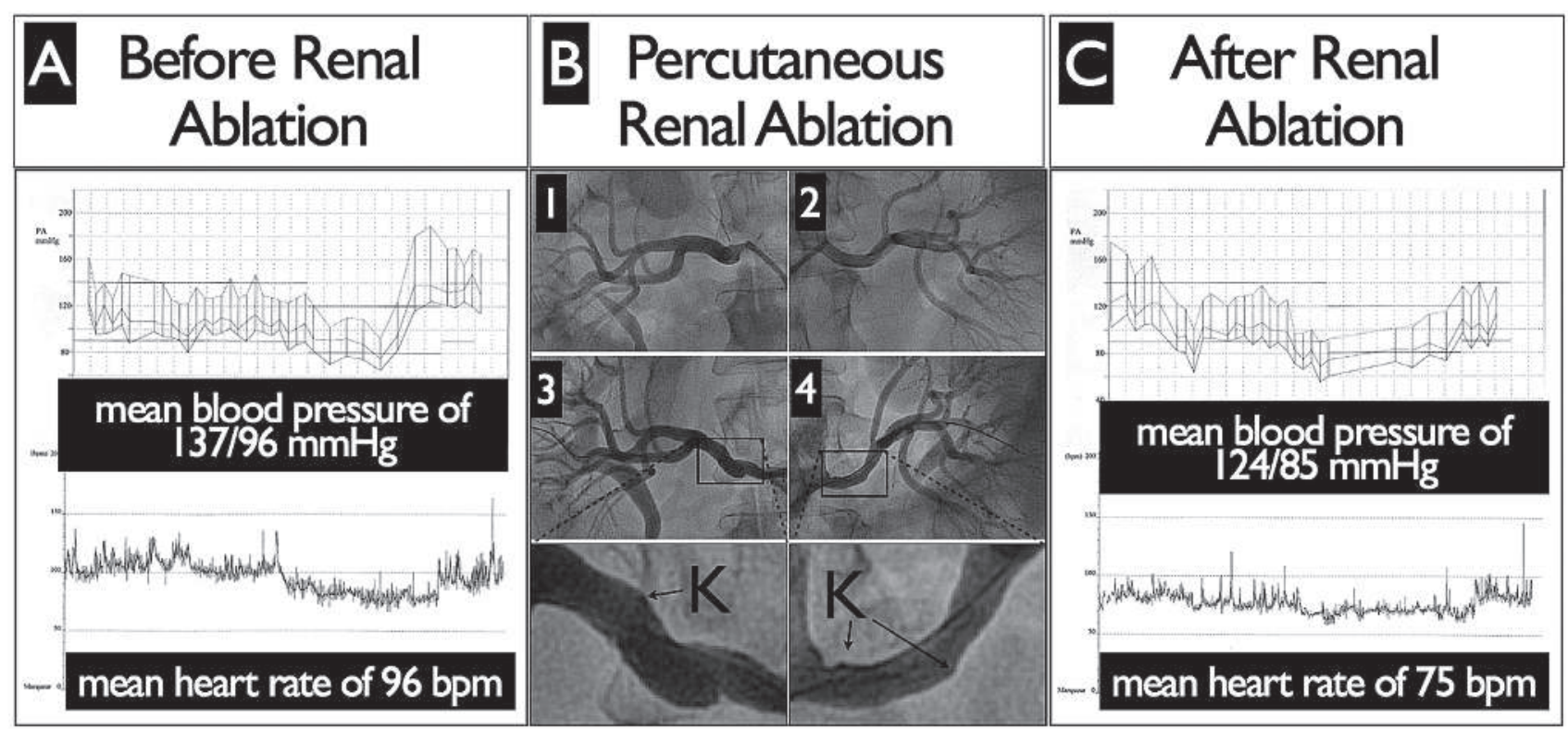

Funding / potential competing interests: No financial support and no other potential conflict of interest relevant to this article were reported.
Correspondence:

Professor Jean-Jacques Goy, MD

Department of Cardiology

University \& Hospital Fribourg

$\mathrm{CH}-1708$ Fribourg

Switzerland

jjgoy[at]goyman.com 
Briefly, six, 2-min low-power RF ablations were performed within the main stem of each renal artery with the first ablation spot being located distally and subsequent ablations performed by moving proximally in the vessel lumen. Renal angiograms were performed before and after (fig. 1, panel B) the procedure. Post-procedural renal angiograms demonstrated ablation's nodes $(\mathrm{K})$. The outcome of this procedure was uneventful and the patient was discharged on the same medication.

Two weeks after the procedure, 24-hour recordings were repeated and are depicted in figure 1, panel C. In comparison to baseline values, a significant drop in mean blood pressure at 124/85 $\mathrm{mm} \mathrm{Hg}$ was noted on 24-hour monitoring. This was furthermore associated with concomitant decrease in mean heart rate to 75 bpm.

Dysregulation of the autonomic nervous system is implicated in the pathophysiology of essential hyper- tension, metabolic insulin resistance, sleep apnoea syndrome and sinus tachycardia [1,2]. Catheter-based renal sympathetic denervation is a viable option to treat patients with refractory arterial hypertension and has been shown to impact the progression of metabolic insulin resistance and sleep apnoea syndrome. Yet, a reduction of resting heart rate is expected and might be used as early marker of procedural success after percutaneous renal sympathetic denervation.

Key words: renal denervation; hypertension; ablation; renal innervation

\section{References}

1 Scherrer U, Sartori C. Insulin as a vascular and sympathoexcitatory hormone: Implications for blood pressure regulation, insulin sensitivity, and cardiovascular morbidity. Circulation. 1997;96:4104-13.

2 Smith RP, Veale D, Pepin JL, Levy PA. Obstructive sleep apnoea and the autonomic nervous system. Sleep Med Rev. 1998;2:69-92. 